\title{
ON FREDHOLM OPERATORS IN QUARTER-PLANE TOEPLITZ ALGEBRAS
}

\author{
XINHUI JIANG
}

(Communicated by Palle E. T. Jorgensen)

\begin{abstract}
In this note we explicitly construct a Fredholm operator of index one in any skew quarter-plane Toeplitz algebra and determine the $K$-theory of related $C^{*}$-algebras.
\end{abstract}

In this note we study the existence of Fredholm operators of any given index in the Toeplitz algebra on a skew quarter-plane, which was previously introduced and studied by Douglas and Howe ([1]), and Park ([5] and [6]).

The Hilbert space $\ell^{2}\left(\mathbb{Z}^{2}\right)$ has a canonical basis $\left\{\chi_{(s, t)}:(s, t) \in \mathbb{Z}^{2}\right\}$, where each $\chi_{(s, t)}$ is the obvious characteristic function on $\mathbb{Z}^{2}$. There is a natural unitary representation $M$ of $\mathbb{Z}^{2}$ on the space, given by translation:

$$
M_{(m, n)}\left(\chi_{(s, t)}\right)=\chi_{(s+m, t+n)} .
$$

Any two different lines $y=\alpha \cdot x$ and $y=\beta \cdot x$ on $\mathbb{R}^{2}$ cut the plane into four cone-like pieces. We take any piece and call it $\mathscr{P}$. For convenience we assume that $\mathscr{P}$ includes its boundary. Then $\mathscr{P}$ determines a closed subspace $\mathscr{H}$ of $\ell^{2}\left(\mathbb{Z}^{2}\right)$, spanned by all elements of the form $\chi_{(s, t)}$ where $(s, t) \in \mathscr{P}$. The Toeplitz algebra $\mathscr{T}$ for $\mathscr{P}$ is defined to be the $C^{*}$-algebra on $\mathscr{H}$ generated by the compressions of $M_{(m, n)}$ 's on $\mathscr{H}$. See [5], [6] for more details.

In [5], [6], E. Park gives a detailed study of the index theory for such algebras. He shows, among other things, that there is a Fredholm operator of any given index in the algebra when at least one of $\alpha, \beta$ is a rational number. This helps to give a complete analysis in that situation, including the calculation of the $K$-theory of certain related algebras (cf. [5], [6] and [7]).

The purpose of this note is to deal with the remaining situation, when both slopes are irrational, although our approach works in general, regardless of the rationality of the slopes. The main result is the following:

Main Theorem. $\mathscr{T}$ contains Fredholm operators of any given index.

Received by the editors December 2, 1993 and, in revised form, February 24, 1994.

1991 Mathematics Subject Classification. Primary 47B35; Secondary 46L80.

$K e y$ words and phrases. Skew quarter-plane Toeplitz algebra, Fredholm operator, $K$-theory. 
In fact we have a slightly stronger result (Theorem 1): the Fredholm operators we construct are in the unitification of some commutator ideals. This will be needed to determine the $K$-theory of these commutator ideals.

The rest of this note is organized as follows: We first reduce the situation to the special case where $0<\alpha \leq 1 / 2$ and $1 \leq \beta<+\infty$. Then in this special case we take the piece $\mathscr{P}$ in the first quadrant, and explicitly construct a Fredholm operator of index one. Finally, using Theorem 1 we calculate the $K$-theory of some related $C^{*}$-algebras.

The higher dimensional analogy of this study appears to be more challenging. Although some structural results of the $C^{*}$-algebras involved are known, we have not been able to decide their Fredholm theory and $K$-theory in general.

We would like to thank Professor Ronald Douglas and the referee for their helpful comments on earlier versions of this paper.

\section{1}

In this section we will consider the action of the group $G L(2, \mathbb{Z})$ on a pair of lines through the origin in $\mathbb{R}^{2}$. If a line through the origin has $s$ as its slope, the slope of its image under $g \in G L(2, \mathbb{Z})$ will be called $g(s)$. Given two different lines through the origin, with slopes $\alpha, \beta$ respectively (the slope of the vertical axis is denoted by " $\infty$ "), we will show in this section that there is a $g \in G L(2, \mathbb{Z})$ such that $0<g(\alpha) \leq 1 / 2$ and $1 \leq g(\beta)<+\infty$.

This has some implications for the corresponding Toeplitz algebras. Note that if a transformation $g \in G L(2, \mathbb{Z})$ maps $\mathscr{P}$ onto $\mathscr{P} \prime$, then $g$ induces a unitary operator $U_{g}$ between $\mathscr{H}$ and $\mathscr{H}^{\prime}$, and $\mathscr{T}$ and $\mathscr{T}^{\prime}$ are spatially isomorphic through $U_{g}$, so they have the same Fredholm index theory. Therefore, in the following sections, we can always assume that

$$
0<\alpha \leq \frac{1}{2} \text { and } 1 \leq \beta<+\infty
$$

without losing any generality.

Step 1: Reduction to the case where $0<\alpha<\beta$. Consider a transformation of the following form:

$$
g_{n}=\left(\begin{array}{cc}
1 & n \\
0 & 1
\end{array}\right), \quad 0<n \in \mathbb{N} .
$$

Note that $g_{n}(\infty)=1 / n$, and $g_{n}(s)=s /(1+n \cdot s)$, if $s \neq \infty$ and $1+n \cdot s \neq 0$. Therefore, for any two given lines, we can always find an integer $n$ large enough so that the slopes of their images under $g$ are very small positive numbers. Therefore, from now on, we assume that $0<\alpha<\beta<+\infty$.

Step 2: Reduction to the case where $0<\alpha \leq 1 / 2$ and $1 \leq \beta<+\infty$. For this purpose we choose a pair $(p, q)$ of positive, relatively-prime integers such that $q / p$ is very close to $\beta$ : if $\beta$ is rational, let $(p, q)$ be the pair determined by $\beta=q / p$; if $\beta$ is irrational, we choose, through continued fractions (cf. [2]), such a pair $(p, q)$ satisfying the following weaker condition:

$$
\alpha<\frac{q}{p} \text { and } 0 \leq \beta-\frac{q}{p}<\frac{1}{p^{2}} .
$$

Note that for such a pair, we have

$$
\frac{\beta-\alpha}{q-p \cdot \alpha}=\frac{1}{p} \cdot \frac{\beta-\alpha}{q / p-\alpha} \geq \frac{1}{p}>p \cdot \beta-q .
$$


Since $p, q$ are relatively prime, we can find a pair $(m, n)$ of integers such that $m \cdot p+n \cdot q=1$. Moreover, we can choose such a pair which satisfies the following additional condition:

$$
0<\frac{m+n \cdot \alpha}{q-p \cdot \alpha} \leq 1
$$

This can be done since, if $m \cdot p+n \cdot q=1$, then $(m-k \cdot q) \cdot p+(n+k \cdot p) \cdot q=1$ for any integer $k$, and

$$
\frac{(m-k \cdot q)+(n+k \cdot p) \cdot \alpha}{q-p \cdot \alpha}=\frac{m+n \cdot \alpha}{q-p \cdot \alpha}-k .
$$

Therefore, we can choose $(m, n)$ so that (3) is satisfied.

Now consider the following transformation:

$$
g=\left(\begin{array}{cc}
m+q & n-p \\
m & n
\end{array}\right) \in S L(2, \mathbb{Z}) .
$$

We claim that $0<g(\alpha) \leq 1 / 2$. Indeed,

$$
g(\alpha)=\frac{m+n \cdot \alpha}{(m+q)+(n-p) \cdot \alpha}=\frac{m+n \cdot \alpha}{(m+n \cdot \alpha)+(q-p \cdot \alpha)} .
$$

But since $q-p \cdot \alpha>0$, (2) implies $0<m+n \cdot \alpha \leq q-p \cdot \alpha$, and therefore, $0<g(\alpha) \leq 1 / 2$.

Next we consider the image of $y=\beta \cdot x$ under $g$. Again the new slope is

$$
g(\beta)=\frac{m+n \cdot \beta}{(m+n \cdot \beta)+(q-p \cdot \beta)} .
$$

Note that in this case, we have

$$
\begin{aligned}
m+n \cdot \beta & >(m+n \cdot \beta)+(p \cdot \beta-q) \cdot\left(\frac{m+n \cdot \alpha}{q-p \cdot \alpha}-1\right) \quad(\text { by }(3)) \\
& =\frac{(m+n \cdot \beta)(q-p \cdot \alpha)+(p \cdot \beta-q)(m+n \cdot \alpha)}{q-p \cdot \alpha}-(p \cdot \beta-q) \\
& =\frac{\beta-\alpha}{q-p \cdot \alpha}-(p \cdot \beta-q) \\
& >0 \quad \text { (by }(2)) .
\end{aligned}
$$

Note that if $\beta$ is rational, then by our choice of $p$ and $q$, it follows that $q-p \cdot \beta=0$, and therefore, $g(\beta)=1$. If $\beta$ is irrational, then $(m+n \cdot \beta)+$ $(q-p \cdot \beta) \neq 0$, and there are two possibilities:

Case 1: $(m+n \cdot \beta)+(q-p \cdot \beta)>0$. In this case, since $q-p \cdot \beta<0$, we have

$$
0<(m+n \cdot \beta)+(q-p \cdot \beta)<(m+n \cdot \beta),
$$

and hence $1<g(\beta)<+\infty$. This is exactly what we want.

Case 2: $(m+n \cdot \beta)+(q-p \cdot \beta)<0$. In this case $g(\beta)$ is a negative irrational number. Therefore there is a unique integer $N \geq 1$ such that

$$
-\frac{1}{N-1}<g(\beta)<-\frac{1}{N} \text {. }
$$


(When $N=1$, this means $g(\beta)<-1$.) Then we consider the images of $y=g(\alpha) \cdot x$ and $y=g(\beta) \cdot x$ under the following transformation $g_{N}$ :

$$
g_{N}=\left(\begin{array}{cc}
1 & N \\
0 & 1
\end{array}\right) .
$$

The slope of the image of $y=g(\alpha) \cdot x$ under $g_{N}$ is $g(\alpha) /(1+N \cdot g(\alpha))$, which is between 0 and $1 / 2$ for any $N \geq 1$, since $0<g(\alpha)<1 / 2$. And the slope of the image of $y=g(\beta) \cdot x$ under $g_{N}$ is $g(\beta) /(1+N \cdot g(\beta))$, which can be shown to be bigger than 1 since, for $x<0$,

$$
\frac{x}{1+N \cdot x}>1 \text { if and only if }-\frac{1}{N-1}<x<-\frac{1}{N} \text {. }
$$

This completes the reduction.

From now on, without any loss of generality, we will assume that condition (†) holds; that is, $0<\alpha \leq 1 / 2$ and $1 \leq \beta<+\infty$.

Now we briefly review some constructions from [5], [6], to which we refer for details. For convenience, we make the following conventions: If $A$ is a subset of $\mathbb{R}$, then $A_{+}=A \cap[0,+\infty)$ and $A_{-}=A \cap(-\infty, 0]$. If $A, B$ are subsets of $\mathbb{R}$, then $\mathscr{H}(A \times B)$ is the closed subspace of $\ell^{2}\left(\mathbb{Z}^{2}\right)$ spanned by $\left\{\chi_{(m, n)}: n-m \cdot \alpha \in A, n-m \cdot \beta \in B\right\}$, and $P(A \times B)=$ the orthogonal projection onto $\mathscr{H}(A \times B)$. For the empty set $\varnothing$, we define $\mathscr{H}(\varnothing)=\{0\}$ and $P(\varnothing)=0$.

Recall from [1], [5] and [6] that:

$$
\begin{aligned}
& \mathscr{H}^{\alpha}=\mathscr{H}([0,+\infty) \times \mathbb{R}) ; \quad P^{\alpha}=P([0,+\infty) \times \mathbb{R}), \\
& \mathscr{H}^{\beta}=\mathscr{H}(\mathbb{R} \times(-\infty, 0]) ; \quad P^{\beta}=P(\mathbb{R} \times(-\infty, 0]), \\
& \mathscr{H}^{\alpha, \beta}=\mathscr{H}^{\alpha} \cap \mathscr{H}^{\beta}=\mathscr{H}([0,+\infty) \times(-\infty, 0]) ; \\
& P^{\alpha, \beta}=P^{\alpha} \cdot P^{\beta}=P([0,+\infty) \times(-\infty, 0]) .
\end{aligned}
$$

Definition 1 (cf. [1], [5], [6]). For $\sharp=\alpha, \beta$, or $(\alpha, \beta)$, let $\mathscr{T}^{\sharp}$ be the $C^{*}$ algebra on $\mathscr{H}^{\sharp}$ generated by the compressions of $\left\{M_{(m, n)}\right\}$ 's on $\mathscr{H}^{\sharp}$, and $\mathscr{C}^{\sharp}$ the commutator ideal of $\mathscr{T}^{\sharp}$.

It will be useful for us to take a closer look at these algebras.

Note that $P([a, b) \times \mathbb{R})$ is in $\mathscr{T}^{\alpha}$ for any $a, b \in\{\mathbb{Z}+\mathbb{Z} \cdot \alpha\}_{+} \cup\{+\infty\}$. Indeed, if $a=n-m \cdot \alpha \geq 0, b=t-s \cdot \alpha \geq 0$ and $a \leq b$, then

$$
\begin{gathered}
P([a,+\infty) \times \mathbb{R})=P^{\alpha} M_{(m, n)} P^{\alpha} M_{(-m,-n)} P^{\alpha} \in \mathscr{T}^{\alpha}, \\
P([b,+\infty) \times \mathbb{R})=P^{\alpha} M_{(s, t)} P^{\alpha} M_{(-s,-t)} P^{\alpha} \in \mathscr{T}^{\alpha},
\end{gathered}
$$

and

$$
P([a, b) \times \mathbb{R})=P([a,+\infty) \times \mathbb{R})-P([b,+\infty) \times \mathbb{R}) \in \mathscr{T}^{\alpha} .
$$

Moreover, if $M_{(m, n)}$ maps $\mathscr{H}([a, b) \times \mathbb{R})$ into $\mathscr{H}^{\alpha}$ (that is, if $a+(n-m \cdot \alpha) \geq$ $0)$, then

$$
M_{(m, n)} P([a, b) \times \mathbb{R})=P^{\alpha} M_{(m, n)} P([a, b) \times \mathbb{R}) P^{\alpha} \in \mathscr{T}^{\alpha} .
$$

We claim that the set $\mathscr{G}^{a}$ of operators of the form $M_{(m, n)} P([a, b) \times \mathbb{R})$, where $a, b \in\{\mathbb{Z}+\mathbb{Z} \cdot \alpha\}_{+} \cup\{+\infty\}$ and $a+(n-m \cdot \alpha) \geq 0$, is a total set in 
$\mathscr{T}^{\alpha}$. This follows from the following basic identity: for any $(m, n) \in \mathbb{Z}^{2}$ and any $a \in \mathbb{R}$,

$$
P([a,+\infty) \times \mathbb{R}) M_{(m, n)}=M_{(m, n)} P([a-(n-m \alpha),+\infty) \times \mathbb{R})
$$

as operators on $\ell^{2}\left(\mathbb{Z}^{2}\right)$. From $(\ddagger)$ it is easy to show that $P^{\alpha} M_{(m, n)} P^{\alpha} \in \mathscr{G}^{\alpha}$ for any $(m, n) \in \mathbb{Z}^{2}$ and $\mathscr{G}^{\alpha} \cdot \mathscr{G}^{\alpha} \subseteq \mathscr{G}^{\alpha}$. Therefore $\mathscr{G}^{\alpha}$ is a total set in $\mathscr{T}^{\alpha}$.

Similarly, let $\mathscr{G}_{c}^{\alpha}$ be the set of operators of the form $M_{(m, n)} P([a, b) \times \mathbb{R})$, where $a, b \in\{\mathbb{Z}+\mathbb{Z} \cdot \alpha\}_{+}$and $a+(n-m \cdot \alpha) \geq 0$. Again it follows from ( $\left.\ddagger\right)$ that $\left[\mathscr{G}^{\alpha}, \mathscr{G}^{\alpha}\right] \subseteq \mathscr{G}_{c}^{\alpha}-\mathscr{G}_{c}^{\alpha}, \mathscr{G}^{\alpha} \cdot \mathscr{G}_{c}^{\alpha} \subseteq \mathscr{G}_{c}^{\alpha}$, and $\mathscr{G}_{c}^{\alpha} \cdot \mathscr{G}^{\alpha} \subseteq \mathscr{G}_{c}^{\alpha}$. So $\mathscr{G}_{c}^{\alpha}$ is total in $\mathscr{C}^{\alpha}$.

We also have similar descriptions for $\mathscr{T}^{\beta}, \mathscr{C}^{\beta}, \mathscr{T}^{\alpha, \beta}$ and $\mathscr{C}^{\alpha, \beta}$. Let us be a little more specific about the latter two algebras. form

A careful inspection reveals that the set $\mathscr{G}$ of operators on $\mathscr{H}^{\alpha, \beta}$ of the

$$
M_{(m, n)} P([a, b) \times(c, d]),
$$

where

$$
a, b \in\{\mathbb{Z}+\mathbb{Z} \cdot \alpha\}_{+} \cup\{+\infty\}, \quad c, d \in\{\mathbb{Z}+\mathbb{Z} \cdot \beta\}_{-} \cup\{-\infty\},
$$

and

$$
a+(n-m \cdot \alpha) \geq 0, \quad d+(n-m \cdot \beta) \leq 0,
$$

is a total subset of $\mathscr{T}^{\alpha, \beta}$. And in $\mathscr{C}^{\alpha, \beta}$, the set

$\mathscr{G}_{c}=\left\{M_{(m, n)} P([a, b) \times(c, d]) \in \mathscr{G}\right.$ : at least one of $b, c$ is a finite number $\}$

is a total subset. We will have more to say about these and some other algebras later. But now we are ready to construct a Fredholm operator $T$ of index -1 . Set

$$
T=1-P\left([0,1) \times \mathbb{R}_{-}\right)+M_{(1,1)} \cdot P\left([0, \alpha) \times \mathbb{R}_{-}\right)+M_{(1,0)} \cdot P\left([\alpha, 1) \times \mathbb{R}_{-}\right) .
$$

Theorem 1. $T-1 \in \mathscr{C}^{\alpha, \beta}$ and $\operatorname{Index}\left(T^{*}\right)=1$.

Proof. By our description of $\mathscr{C}^{\alpha, \beta}$, obviously $T-1$ is in $\mathscr{C}^{\alpha, \beta}$.

Note that by condition $(\dagger)$, if $\chi_{(m, n)} \in \mathscr{H}^{\alpha, \beta}$, then $m \geq 0$ and $n \geq 0$.

Claim (I). $T$ is an isometry.

Case (I.1): When $\chi_{(m, n)} \in \mathscr{H}\left([0, \alpha) \times \mathbb{R}_{-}\right)$, that is, when

$$
0 \leq n-m \cdot \alpha<\alpha, \quad n-m \cdot \beta \leq 0,
$$

then

$$
1-\alpha \leq(n+1)-(m+1) \cdot \alpha<1, \quad(n+1)-(m+1) \cdot \beta=(n-m \cdot \beta)+(1-\beta) \leq 0 .
$$

Therefore, $T$ sends $\mathscr{H}\left([0, \alpha) \times \mathbb{R}_{-}\right)$isometrically into $\mathscr{H}\left([1-\alpha, 1) \times \mathbb{R}_{-}\right)$.

Case (I.2): When $\chi_{(m, n)} \in \mathscr{H}\left([\alpha, 1) \times \mathbb{R}_{-}\right)$, that is, when

$$
\alpha \leq n-m \cdot \alpha<1, \quad n-m \cdot \beta \leq 0,
$$

then

$$
0 \leq n-(m+1) \cdot \alpha<1-\alpha, \quad n-(m+1) \cdot \beta=(n-m \cdot \beta)-\beta \leq 0 .
$$

Therefore, $T$ sends $\mathscr{H}\left([\alpha, 1) \times \mathbb{R}_{-}\right)$isometrically into $\mathscr{H}\left([0,1-\alpha) \times \mathbb{R}_{-}\right)$.

Since $\mathscr{H}\left([1-\alpha, 1) \times \mathbb{R}_{-}\right)$and $\mathscr{H}\left([0,1-\alpha) \times \mathbb{R}_{-}\right)$are orthogonal, combining Cases (I.1) and (I.2), we know that $T$ maps $\mathscr{H}\left([0,1) \times \mathbb{R}_{-}\right)$isometrically into 
itself. On the other hand, by definition $T=1$ on $\mathscr{H}\left([1,+\infty) \times \mathbb{R}_{-}\right)$. So $T$ is an isometry on $\mathscr{H}^{\alpha, \beta}$.

Claim (II). Kernel $\left(T^{*}\right)=\left\{\mathbb{C} \cdot \chi_{(0,0)}\right\}$.

Case (II.1): $\chi_{(m, n)} \in \mathscr{H}\left([1-\alpha, 1) \times \mathbb{R}_{-}\right)$. In this case,

$$
1-\alpha \leq n-m \cdot \alpha<1 \text { and } n-m \cdot \beta \leq 0 \text {, }
$$

and therefore,

$$
0 \leq(n-1)-(m-1) \cdot \alpha<\alpha, \quad(n-1)-(m-1) \cdot \beta=(n-m \cdot \beta)-(1-\beta) .
$$

So $\chi_{(m, n)} \in \operatorname{Range}(T)$, unless $n-m \cdot \beta>1-\beta$. But if $n-m \cdot \beta>1-\beta$, then

$$
m \cdot \beta<\beta-1+n<m \cdot \alpha+\beta,
$$

since $n-m \cdot \alpha<1$. Hence $m<\beta /(\beta-\alpha)$, and we have $\beta /(\beta-\alpha) \leq 2$ since $1 \leq \beta<+\infty$ and $0<\alpha \leq 1 / 2$. So $m=0,1$.

But $m=0$ is contradictory to the condition $1-\alpha \leq n-m \cdot \alpha<1$. And when $m=1$, the same condition implies that $n=1$. But $\chi_{(1,1)}$ is in $\operatorname{Range}(T)$ since it is the image of $\chi_{(0,0)}$ under $T$.

So we have proved that $\operatorname{Range}(T)$ contains $\mathscr{H}\left([1-\alpha, 1) \times \mathbb{R}_{-}\right)$.

Case (II.2): $\chi_{(m, n)} \in \mathscr{H}\left([0,1-\alpha) \times \mathbb{R}_{-}\right)$. In this case,

$$
0 \leq n-m \cdot \alpha<1-\alpha, \quad n-m \cdot \beta \leq 0,
$$

and therefore,

$$
\alpha \leq n-(m-1) \cdot \alpha<1, \quad n-(m-1) \cdot \beta=(n-m \cdot \beta)+\beta .
$$

So $\chi_{(m, n)} \in \operatorname{Range}(T)$, unless $0 \geq n-m \cdot \beta>-\beta$. But if $n-m \cdot \beta>-\beta$, then

$$
(m-1) \cdot \beta<n<(m-1) \cdot \alpha+1,
$$

since $n-m \cdot \alpha<1-\alpha$. Hence $(m-1)<1 /(\beta-\alpha)$, and we have $1 /(\beta-\alpha) \leq 2$ since $\beta-\alpha \geq 1 / 2$. So $m=0,1,2$.

When $m=0$, the condition $0 \leq n-m \cdot \alpha<1-\alpha$ implies $n=0$. And, it is easy to see that $\chi_{(0,0)}$ is not in the range of $T$.

On the other hand, $m=1$ is contradictory to the condition $0 \leq n-m \cdot \alpha<$ $1-\alpha$.

When $m=2$, the same condition implies that $n=1$. But $\chi_{(2,1)}$ is in Range $(T)$ since it is the image of $\chi_{(1,1)}$ under $T$.

Combining these two cases, we know that $\operatorname{Range}(T)$ contains the orthogonal complement of $\left\{\mathbb{C} \cdot \chi_{(0,0)}\right\}$ in $\mathscr{H}\left([0,1) \times \mathbb{R}_{-}\right)$. On the other hand, $\operatorname{Range}(T)$ certainly contains $\mathscr{H}\left([1,+\infty) \times \mathbb{R}_{-}\right)$, and therefore, $\operatorname{Kernel}\left(T^{*}\right)=\left\{\mathbb{C} \cdot \chi_{(0,0)}\right\}$.

Finally, index $\left(T^{*}\right)=\operatorname{dim}\left(\operatorname{Ker}\left(T^{*}\right)\right)-\operatorname{dim}(\operatorname{Ker}(T))=1-0=1$. This finishes the proof.

The main theorem of this note (see $\S 0$ ) follows readily from Theorem 1.

Remarks. (1) Given two lines through the origin whose slopes satisfy condition $(\dagger)$, to define a Toeplitz algebra we need to choose one piece from the four skew quarter-planes. Our construction corresponds to the one in the first quadrant. We can also choose another piece and construct a Toeplitz algebra in the same way. Our recipe for producing a Fredholm operator still works, and we get a Fredholm operator whose index is either 1 or -1 . More generally, for any 
two different lines through the origin, similar constructions still work. In fact, if $0<\alpha<1$, then the same recipe in $\S 2$ produces a Fredholm operator in the Toeplitz algebra, whose index should be either 1 or -1 from $K$-theoretic considerations. However, even in this case, without condition $(t)$ it seems difficult to calculate the index directly (by comparing the numbers of integer pairs in certain regions).

(2) Park [5], [6] has used cyclic cohomology to give a formula for computing the index of Fredholm operators in quarter-plane Toeplitz algebras, which is valid in general, even when both $\alpha$ and $\beta$ are irrational.

3

Following [5], [6] and [7], we now examine the implications of Theorem 1 on the $K$-theory of certain related algebras. We will only discuss the case where both $\alpha$ and $\beta$ are irrational, and refer the reader to [5], [6] and [7] for the other cases.

According to [5], [6], $\mathscr{T}^{\alpha, \beta}$ contains all compact operators on $\mathscr{H}^{\alpha, \beta}$; therefore, there is a $C^{*}$-algebra exact sequence

$$
0 \rightarrow \mathscr{K} \rightarrow \mathscr{T}^{\alpha, \beta} \rightarrow \mathscr{S}^{\alpha, \beta} \rightarrow 0 .
$$

By identifying $\mathscr{S}^{\alpha, \beta}$ in a more transparent way and using the Mayer-Vietoris sequence, Park[5] [6] shows that $K_{0}\left(\mathscr{S}^{\alpha, \beta}\right) \cong \mathbb{Z}^{3}$ and $K_{1}\left(\mathscr{S}^{\alpha, \beta}\right) \cong \mathbb{Z}$. Together with the construction of the Fredholm operator $T^{*}$ in $\mathscr{T}^{\alpha, \beta}$ of index one in $\S 2$, this $K$-theoretic calculation implies (cf. [5], [6])

Theorem 2. The index is a complete stable deformation invariant for Fredholm operators in $\mathscr{T}^{\alpha, \beta}$.

Now we look at the six-term exact sequence in $K$-theory that arises from (4):

$$
\begin{gathered}
\mathbb{Z} \cong K_{0}(\mathscr{K}) \longrightarrow K_{0}\left(\mathscr{T}^{\alpha, \beta}\right) \longrightarrow K_{0}\left(\mathscr{S}^{\alpha, \beta}\right) \cong \mathbb{Z}^{3} \\
\quad \uparrow_{\text {index map }} \longrightarrow K_{1}(\mathscr{K}) \cong\{0\} .
\end{gathered}
$$

Since we have a Fredholm operator $T \in \mathscr{T}^{\alpha, \beta}$ which has index -1 , it is easy to see that the index map in the exact sequence is an isomorphism; therefore,

$$
K_{0}\left(\mathscr{T}^{\alpha, \beta}\right) \cong K_{0}\left(\mathscr{S}^{\alpha, \beta}\right) \cong \mathbb{Z}^{3}, \quad K_{1}\left(\mathscr{T}^{\alpha, \beta}\right) \cong\{0\}
$$

If we restrict the exact sequence (4) to $\mathscr{C}^{\alpha, \beta}$, we get another exact sequence, which can be identified (cf. [5]) more clearly as

$$
0 \rightarrow \mathscr{K} \rightarrow \mathscr{C}^{\alpha, \beta} \rightarrow \mathscr{C}^{\alpha} \oplus \mathscr{C}^{\beta} \rightarrow 0 .
$$

Then since $T-1 \in \mathscr{C}^{\alpha, \beta}$, the index map in the following exact sequence is onto:

$$
\begin{aligned}
& \mathbb{Z} \cong K_{0}(\mathscr{K}) \quad \longrightarrow K_{0}\left(\mathscr{C}^{\alpha, \beta}\right) \longrightarrow K_{0}\left(\mathscr{C}^{\alpha}\right) \oplus K_{0}\left(\mathscr{C}^{\beta}\right) \\
& \uparrow \text { index map } \\
& K_{1}\left(\mathscr{C}^{\alpha}\right) \oplus K_{1}\left(\mathscr{C}^{\beta}\right) \longleftarrow K_{1}\left(\mathscr{C}^{\alpha, \beta}\right) \longleftarrow K_{1}(\mathscr{K}) \cong\{0\} .
\end{aligned}
$$


It is known (cf. [3], [4]) that $K_{0}\left(\mathscr{C}^{\alpha}\right) \cong K_{0}\left(\mathscr{C}^{\beta}\right) \cong \mathbb{Z}^{2}$ and $K_{1}\left(\mathscr{C}^{\alpha}\right) \cong K_{1}\left(\mathscr{C}^{\beta}\right) \cong$ $\mathbb{Z}$, and therefore,

$$
K_{0}\left(\mathscr{C}^{\alpha, \beta}\right) \cong \mathbb{Z}^{4}, \quad K_{1}\left(\mathscr{C}^{\alpha, \beta}\right) \cong \mathbb{Z} .
$$

Finally we consider a subalgebra of $\mathscr{T}^{\alpha, \beta}$. Recall from $\S_{2}$ that $\mathscr{G}_{c}$ is a total set in $\mathscr{C}^{\alpha, \beta}$. Let us consider the subalgebra $\mathscr{J}^{\alpha}$ of $\mathscr{C}^{\alpha, \beta}$ generated by the following subset of $\mathscr{G}_{c}$ :

$$
\left\{M_{(m, n)} P([a, b) \times(c, d]) \in \mathscr{G}: b \neq+\infty\right\} .
$$

It is the $C^{*}$-algebra on $\mathscr{H}^{\alpha, \beta}$ generated by the compressions on $\mathscr{H}^{\alpha, \beta}$ of operators in $\mathscr{C}^{\alpha} \subseteq \mathscr{L}\left(\mathscr{H}^{\alpha}\right)$. Crucial for our purpose is the fact that for the Fredholm operator $T$ we constructed in $\S 2, T-1 \in \mathcal{J}^{\alpha}$.

Restricting the sequence (4) to $\mathcal{J}^{\alpha}$, we get another short exact sequence of $C^{*}$-algebras (cf. [5], [6]):

$$
0 \rightarrow \mathscr{K} \rightarrow \mathscr{J}^{\alpha} \rightarrow \mathscr{C}^{\alpha} \rightarrow 0
$$

and its corresponding six-term exact sequence in $K$-theory:

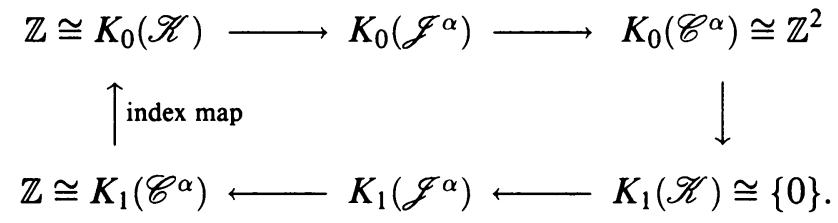

For the same reason as above, the index map is an isomorphism, and it follows that

$$
K_{0}\left(\mathcal{J}^{\alpha}\right) \cong \mathbb{Z}^{2}, \quad K_{1}\left(\mathcal{J}^{\alpha}\right) \cong\{0\} .
$$

\section{REFERENCES}

1. R. G. Douglas and R. Howe, On the $C^{*}$-algebra of Toeplitz operators on the quarter-plane, Trans. Amer. Math. Soc. 158 (1971), 203-217.

2. G. H. Hardy and E. M. Wright, An introduction to the theory of numbers, 4th ed., Oxford Univ. Press, London, 1975.

3. R. Ji and J. Kaminker, The K-theory of Toeplitz extensions, J. Operator Theory 19 (1988), 347-354.

4. R. Ji and J. Xia, On the classification of commutator ideals, J. Funct. Anal. 78 (1988), 208-232.

5. E. Park, The index theory of Toeplitz algebras on the skew quarter-plane, Ph. D. thesis, SUNY at Stony Brook, Stony Brook, NY, 1988.

6. Index theory and Toeplitz algebras on certain cones in $Z^{2}$, J. Operator Theory 23 (1990), 125-146.

7. E. Park and C. Schochet, On the K-theory of quarter-plane Toeplitz algebras, Internat. J. Math. 2 (1991), 195-204.

Department of Mathematics, SUNY at Stony Brook, Stony Brook, New York 11794 $5 Z 5$

Current address: The Fields Institute, 185 Columbia St. W., Waterloo, Ontario, Canada N2L

E-mail address: jiang@ields.uwaterloo.ca 